\title{
Stress related to COVID-19 pandemic as a trigger for disease activity in multiple sclerosis: a case report
}

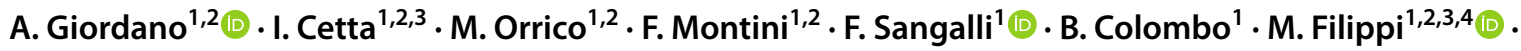 \\ V. Martinelli ${ }^{1}$ (1)
}

Received: 13 March 2021 / Accepted: 24 June 2021 / Published online: 23 July 2021

(c) Fondazione Società Italiana di Neurologia 2021

To the Editor,

Increased incidence of autoimmune and autoinflammatory systemic and neurological diseases following severe acute respiratory syndrome coronavirus 2 (SARS-CoV-2) infection $[1,2]$ has been reported. The biological mechanisms underlying this association may involve virus-related triggering of immune pathways and cytokine storms [1]. In addition, the coronavirus disease 2019 (COVID-19) pandemic has been recognized as a major stressful event and stressful conditions have been independently associated with autoimmune diseases, since they impact on the dysregulation of immune pathways and may interact with the above-cited mechanisms [1]. Stressful conditions are triggers for disease activity in multiple sclerosis (MS) too [3], but whether the stress induced by COVID-19 pandemic may influence disease activity in MS has never been investigated.

\section{Case report}

We report the case of a 65 -year-old man who had been diagnosed with primary progressive MS in 2013 for subtle progressive gait and balance impairment during the previous years. Treatment with methotrexate $(7.5 \mathrm{mg}$ weekly) was started in March 2015, since the patient suffered from psoriasis, too. The patient was not showing any sign of disease

V. Martinelli

martinelli.vittorio@hsr.it

1 Neurology Unit, IRCCS San Raffaele Scientific Institute, Milan, Italy

2 Neurophysiology Unit, IRCCS San Raffaele Scientific Institute, Via Olgettina 48, 20132 Milan, Italy

3 Neuroimaging Research Unit, Division of Neuroscience, IRCCS San Raffaele Scientific Institute, Milan, Italy

4 Vita-Salute San Raffaele University, Milan, Italy activity since 2015, as no new or contrast-enhancing lesions at magnetic resonance imaging (MRI) and no new relapses had been reported over the years. Expanded Disability Status Scale (EDSS) score was 3.5 in February 2016. The latest MRI in January 2020 was stable, when compared to previous examination in 2019 (Fig. 1A).

In February 2020, Italy was hit very hard by SARSCoV-2 pandemic, with the region of Lombardy, where the patient lived, being the most affected area and reporting one of the highest numbers of infections and deaths in Europe. The patient suffered from a consistent amount of stress because of the pandemic and the consequential lockdown, mainly because of major issues he had to face as he was the head of a large technical family business in the region of Lombardy. During the lockdown, he had to shut down his factory and put his employees temporarily out of work. He continued treatment with methotrexate.

After the lockdown, in June 2020, the patient started to complain about depressed mood, apathy, confusion, and progressive cognitive decline with impairment of reasoning, attention, and reading skills. At the same period, his walking impairment rapidly worsened and EDSS was 5.0. For this reason, he was admitted to the hospital and he underwent a brain MRI, which showed more than 40 new hyperintense lesions in T2/fluid-attenuated inversion recovery (FLAIR) sequences. Post-contrast enhancement was observed in most of the lesions (Fig. 1B). Cerebrospinal fluid examination (CSF) was normal, except for a slight increase of lymphomonocytes $(6$ cells $/ \mu \mathrm{L})$. An extensive panel ruled out central nervous system infection (Cryptococcus, Epstein-Barr Virus, Citomegalovirus, Herpes Simplex Virus 1 and 2, Human Immunodeficiency Virus 1 and 2). No oligoclonal bands in CSF were found at isoelectrofocusing. Serum anti-acquaporine- 4 and anti-MOG antibodies were negative. Paraneoplastic screening resulted negative, and cardiac embolism was ruled out by echocardiography examination. Real-time polymerase chain reaction on nasopharyngeal swab, and 
Fig. 1 Brain magnetic resonance imaging evolutions. A MRI in January 2020 showing focal hyperintense lesions on axial FLAIR sequence (left) with no contrast enhancement on T1-weighted sequence after gadolinium administration (right). B MRI control in June 2020 showing several new hyperintense lesions on sagittal FLAIR sequences (left) with post-contrast enhancement at T1-weighted sequences (right). C FLAIR (left) and post-contrast T1 (right) sagittal sequences at MRI examination in July 2020 showing significant improvement after intravenous steroid treatment
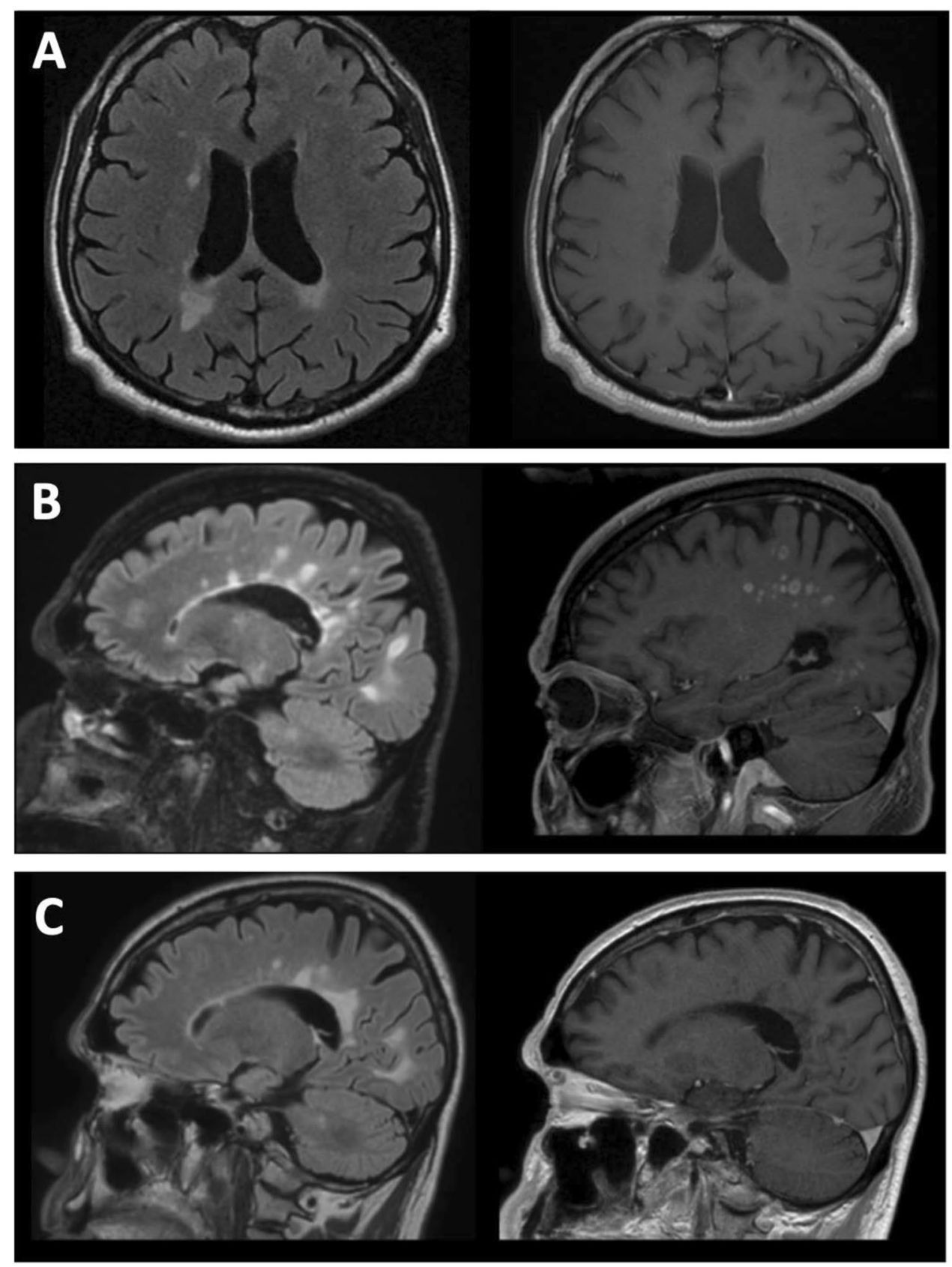

anti-SARS-CoV-2 antibodies through electrochemiluminescence testing were negative, excluding present or past infection. Diagnosis of severe MS relapse was made, and intravenous steroid treatment with methylprednisolone $(1 \mathrm{~g}$ daily for 7 days) was administered, with significant recovery of motor and cognitive performances (EDSS 3.5). A new brain MRI was performed after the end of the intravenous steroid treatment, showing a significant improvement of the radiological findings, as no new T2/FLAIR lesions were reported and contrast enhancement was absent (Fig. 1C). Immediately after steroid, an immunosuppressive treatment with cyclophosphamide was started. At follow-up in
December 2020, MRI examination showed no new signs of disease activity and the patient reported no new symptoms.

\section{Discussion}

We reported the case of a relatively old patient with primary progressive MS, who had stable clinical and MRI evaluations for several years, who was admitted to the hospital for the onset of new neurological symptoms. After ruling out other possible causes, the diagnosis of a severe MS relapse was made, and high-dose steroid treatment was 
administered. The improvement was evident soon after, with dramatic benefit both on the clinical and MRI side.

The patient reported the COVID-19 pandemic as a major stressful event, having a strong negative impact on his personal and professional life. Stress is a well-known trigger for autoimmunity in general and for MS. Major stressful life events have been associated with an increased risk of developing MS and with a higher relapse rate [3]. There are multiple evidences that stressful conditions-like emotional stress, death of a dear one, or exposure to war-may precipitate MS onset and severity [3]. The mechanisms by which stress may play the role of a trigger for MS inflammatory activity are still not clear. Stress is known to lead to the secretion of corticotropin-releasing hormone and neurotensin, which may sustain microglia and mast cell activation [4]. Subsequent blood-brain barrier disruption may be crucial for the migration of autoreactive T-cells and initiation of the autoimmune process, which leads to the formation of new lesions [3]. Another important point is that the relapse in this patient occurred at the end of the lockdown, several weeks after the acute stress related to the pandemic. A similar delay between the onset of the stressful event and the subsequent relapse has been well-described in MS in previous works, leading to the formulation of the "stress resolution hypothesis" [4]. According to this hypothesis, the dissipation of the stress and the subsequent decrease in cortisol levels may facilitate the development of active inflammation, with an increased risk of relapse from weeks to months after the onset of the stress [4].

The neurological implications of COVID-19 seem wide and may reflect both neurodegenerative [5] and autoimmune dysfunctions [1]. Considering the strong negative impact that the pandemic had on the patient and his professional and personal life, we may argue that this severe new MS relapse in a primary progressive course may have been triggered by stress-related mechanisms.

To our knowledge, this is the first reported case of a severe MS relapse possibly related to the stress induced by COVID-19 pandemic.

\section{Conclusions}

Stressful events are well-known triggers for MS relapses. COVID-19 pandemic represents not only a potential lifethreatening condition, but also a major stressful life event and people with MS may be at higher risk of developing new disease activity.

\section{Declarations}

Conflict of interest The authors declare no competing interests.

Ethical approval None

\section{References}

1. Galeotti C, Bayry J (2020) Autoimmune and inflammatory diseases following COVID-19. Nat Rev Rheumatol 16(8):413-414

2. Toscano G, Palmerini F, Ravaglia S et al (2020) GuillainBarré syndrome associated with SARS-CoV-2. N Engl J Med 382(26):2574-2576

3. Artemiadis AK, Anagnostouli MC, Alexopoulos EC (2011) Stress as a risk factor for multiple sclerosis onset or relapse: a systematic review. Neuroepidemiology 36:109-120

4. Mohr DC, Pelletier D (2006) A temporal framework for understanding the effects of stressful life events on inflammation in patients with multiple sclerosis. Brain Behav Immun 20(1):27-36

5. Giordano A, Schwarz G, Cacciaguerra L et al (2020) COVID19: can we learn from encephalitis lethargica? Lancet Neurol 19(7):570

Publisher's note Springer Nature remains neutral with regard to jurisdictional claims in published maps and institutional affiliations. 